\title{
Los insectos galícolas en Schinus fasciculata (Anacardiaceae) en el Espinal del centro de Argentina
}

\author{
Melisa Malcolm', Antonia J. Oggero', Marcelo D. Arana', María del Carmen Tordable' \\ \& Graciela T. Boito 2
}

1. Departamento de Ciencias Naturales, FCEFQyN, Universidad Nacional de Río Cuarto, Ruta 36 Km 601, CP 5800, Córdoba, Argentina.
(melisamalcolm@gmail.com; aoggero@exa.unrc.edu.ar; marana@exa.unrc.edu.ar; mtordable@exa.unrc.edu.ar)
2. Departamento de Biología Agrícola, FAV, Universidad Nacional de Río Cuarto, Ruta 36 Km 601, CP 5800, Córdoba, Argentina. (gboito@ayv.unrc.edu.ar)

ABSTRACT. Galling insects in Schinus fasciculata (Anacardiaceae) in the Espinal from central Argentina. Galls are the most complex interaction between plants and insects during the course of their evolution. The species of galling insects are found in the most of biogeographic regions, but mainly in xeric habitats, as the Espinal eco-region, located in the Pampa Biogeographic Region, Chacoan Subregion. Schinus fasciculata (Griseb.) I. M. Johnst. (Anacardiaceae) it is a tree or shrub, representative from the Espinal eco-region, which supports several kinds of galls. Our objectives are to identify the insect species that induce leaves and stem galls on Schinus fasciculata, in an Espinal relict from province of Córdoba and to describe the exomorphology of galls. In this Espinal relict 18 plants of Schinus fasciculata, in four transects of $100 \mathrm{~m}^{2}$ were selected. Five morphological types of galls were found, three on leaves, induced by insects belonging to Hemiptera Order, and two on stems, induced by insects of Lepidoptera Order. The galling insects were identified at species level and the different morphotypes of galls found were unique to each galling insect species.

KEYWORDS. Córdoba, insect galls, Hemiptera, Lepidoptera, Chacoan Subregion.

RESUMEN. La más compleja de las interacciones que plantas e insectos han desarrollado durante el transcurso de su evolución, son las agallas. Las especies de insectos galícolas se encuentran en la mayoría de las regiones biogeográficas, principalmente en ambientes xéricos, de los cuales un ejemplo lo constituye la ecorregión del Espinal, ubicada en la Provincia Biogeográfica de la Pampa, Subregión Chaqueña. Schinus fasciculata (Griseb.) I.M. Johnst. (Anacardiaceae) es una especie arbórea o arbustiva representativa de la ecorregión del Espinal que presenta diversas agallas entomógenas. Los objetivos del presente trabajo son identificar las especies de insectos que producen agallas en hojas y tallos de Schinus fasciculata en un relicto de Espinal de la provincia de Córdoba y caracterizar exomorfológicamente las agallas. Se seleccionaron 18 ejemplares de S. fasciculata distribuidos en cuatro transectas de $100 \mathrm{~m}^{2}$. Se caracterizaron cinco morfotipos de agallas, tres en hojas, inducidas por insectos del orden Hemiptera y dos en tallos, originadas por insectos del orden Lepidoptera. Los insectos productores de las mismas fueron identificados a nivel de especie y los distintos morfotipos de agallas fueron únicos para cada especie de insecto inductor.

PALABRAS-CLAVE. Córdoba, agallas entomógenas, Hemiptera, Lepidoptera, Subregión Chaqueña.

De todas las interacciones existentes entre plantas e insectos, desarrolladas durante el transcurso de su evolución, la más compleja está representada por la formación de agallas (NIEVES-ALDREy, 1998; SHORTHOUSE et al., 2005), las que son estructuras complejas y simétricas que consisten en un desarrollo anormal (por hiperplasia e hipertrofia) de los tejidos vegetales (NiEves-AldRey, 1998; RAMAN, 2007). La morfología de estas estructuras es única para cada especie de insecto inductor (Cuevas-Reyes et al., 2004), lo que permite diferenciar morfológica, anatómica y fisiológicamente, agallas originadas en una planta por especies relacionadas de insectos (SHORTHOUSE et al., 2005). Los insectos parásitos afectan negativamente a la planta hospedadora por competencia y por disminución del área fotosintética de las hojas, ya que las agallas utilizan recursos que podrían ser destinados para la reproducción de la planta (BARRANCOS et al., 2008).

Las especies de insectos galícolas, que han sido estimadas en 133 mil a nivel mundial (EsPírito-SANTO \& FernANDES, 2007), se encuentran en la mayoría de las regiones biogeográficas, y en la región Neotropical predominan en ambientes xéricos (Fernandes \& Price, 1988, 1992; Price et al., 1998; LARA et al., 2002), siendo los órdenes Diptera, Lepidoptera, Hemiptera, Coleoptera,
Hymenoptera y Thysanoptera los que mayor riqueza de especies galícolas poseen (MANI, 1964). De los ambientes xéricos, un ejemplo lo constituye la ecorregión del Espinal, ubicada en la Provincia Biogeográfica de la Pampa, Subregión Chaqueña (Arana \& Bianco, 2011). El Espinal ocupa el centro-oeste de Argentina, entre los $30^{\circ}$ y $39^{\circ}$ latitud sur, Uruguay y sur del estado brasileño de Rio Grande do Sul (Morrone, 2004) y su vegetación se halla representada por las especies leñosas dominantes Celtis ehrembergiana (Klotzsch) Liebm. (Cannabaceae), Geoffroea decorticans (Gill. ex Hook. \& Arn.) Burkart (Fabaceae) y Schinus fasciculata (Griseb.) I.M. Johnst. (Anacardiaceae). Esta última especie presenta además crecimiento arbustivo, por lo que, de acuerdo a lo expresado por VIEIRA DA Costa et al. (2010), podría alojar un valor intermedio de especies de insectos galícolas, aunque la riqueza de especies galícolas podría también deberse a otras características además de la complejidad estructural, como ser la dinámica de los meristemas, la fisiología del crecimiento y los mecanismos de defensa (ARAúJo \& dos Santos, 2009). Varios autores (Lizer y Trelles \& MolLe, 1945; BurCKHARDT \& BASSET, 2000; BuRCKHARDT, 2004a,b) citan agallas inducidas por insectos de los ordenes Hemiptera y Lepidoptera (Jörgensen, 1917; Costa LimA, 
1945; Fernández \& D’Ambrogio, 2001; Pastrana et al., 2004) en plantas del género Schinus, aunque hay muy escasos antecedentes de insectos galícolas en Schinus fasciculata, una especie estructuralmente compleja, que al habitar ambientes xéricos, sostendría una importante variedad de fauna galícola de la eco-región del Espinal en la provincia de Córdoba.

Considerando la alta diversidad de insectos galícolas en la región Neotropical los estudios de historia natural, riqueza, ecología y dinámica de estas especies continúan siendo escasos y en algunos casos casi nulos, por lo que se requieren mayores estudios que consideren descripciones de los morfotipos de las agallas asociadas a las plantas hospedadoras, así como la identificación de las especies de insectos que las causan. Este tipo de estudios se necesitan con urgencia para lograr una mayor comprensión de la diversidad, distribución, dinámica poblacional y evolución de los insectos galícolas en la región (FERnANDEs et al., 2011, 2014; SANTOS et al., 2012).

De acuerdo a lo expuesto, los objetivos del presente trabajo son identificar las especies de insectos que producen agallas en hojas y tallos de Schinus fasciculata en un relicto de Espinal de la provincia de Córdoba, centro de Argentina, y caracterizar exomorfológicamente las agallas foliares y caulinares producidas en dicha especie.

\section{MATERIALES Y MÉTODOS}

Área de estudio. Reserva Urbana Bosque Autóctono “El Espinal", ubicada aproximadamente entre los $33^{\circ} 10^{\prime} \mathrm{S}$ y $64^{\circ} 20^{\prime} \mathrm{O}$, con una superficie de $7 \mathrm{ha}$, dentro del Campus de la Universidad Nacional de Río Cuarto, Departamento Río Cuarto, Provincia de Córdoba, República Argentina. Se establecieron cuatro transectas de $50 \mathrm{~m}$ por $2 \mathrm{~m} \mathrm{(100}$ $\mathrm{m}^{2}$ ) en dos sitios dentro del área mencionada y en dos fragmentos de bosque nativo próximos a la reserva, una transecta por sitio, respectivamente.

Planta hospedadora. Las plantas de Schinus fasciculata (Griseb.) I.M. Johnst., (Anacardiaceae) son árboles o arbustos que alcanzan hasta $3 \mathrm{~m}$ de altura, poseen ramas grisáceas, glabras, espinescentes; hojas glabras, polimorfas, enteras, cartáceas, fasciculadas, lineal lanceoladas, de ápice obtuso, y cuneadas en la base; inflorescencias en seudorracimos con abundantes flores y brácteas deltoideas con sépalos ovados a oval-lanceolados, y pétalos obovados, glabros (MArtínez CARretero, 2009). Sus frutos son drupas violetas de 3-5 mm de diámetro (BARKLey, 1957). Esta especie se distribuye en Bolivia, Paraguay y en el centro-sur de Argentina hasta Río Negro (Martínez Carretero, 2009). Los ejemplares de esta especie fueron divididos en cuadrantes semejantes lo que permitió que las muestras de tallo y hojas obtenidas fueran representativas.

Los estados inmaduros de los insectos fueron extraídos de agallas disecadas en el laboratorio, desde el mes de septiembre de 2010 hasta abril de 2011, dependiendo de la especie de insecto galícola presente. Los adultos se colectaron mediante bolsas de microtul colocadas a modo de trampa, en ramas terminales, desde el mes de noviembre de 2010 hasta abril de 2011. La frecuencia de colecta fue de tres veces por semana dependiendo de la aparición de cada especie dentro del período de muestreo. Todos los insectos fueron conservados en alcohol al 50\%, con excepción de los microlepidópteros adultos que fueron montados en cajas entomológicas con la técnica de doble montaje y fueron identificados hasta especie mediante el uso de claves, descripciones, bibliografía específica (BURCKHARDT \& Basset, 2000; Burckhardt, 2004a,b; Pastrana et al., 2004), y la consulta a especialistas.

La caracterización exomorfológica de agallas se logró a partir de una muestra de diez agallas tomadas al azar de las plantas, pertenecientes a cada morfotipo encontrado, a las que se les realizaron mediciones con un calibre de $0,02 \mathrm{~mm}$ de precisión, además se registró su ubicación en el órgano de la planta, edad de las ramas sobre las que se situaron, forma, coloración, dehiscencia, agrupación, número de cámaras y número de insectos por cámara.

Las agallas y los insectos inductores fueron observados y caracterizados bajo microscopio estereoscópico modelo Zeiss SV6, y se fotografiaron con cámara digital Canon PowerShot A95.

\section{RESULTADOS}

En las cuatro transectas establecidas dentro del área de estudio, se registraron en total 18 individuos de $S$. fasciculata y cinco tipos morfológicos de agallas, los que se denominaron morfotipos 1, 2 y 3, correspondientes a agallas foliares y morfotipos 4 y 5 , correspondientes a agallas de tallo.

Se encontraron cinco especies de insectos galícolas parasitando a $S$. fasciculata, pertenecientes a tres familias, dos del orden Hemiptera y una del orden Lepidoptera. La ocurrencia de las familias presentó una variación tanto estacional como en abundancia (Tab. I). Al orden Hemiptera pertenecen Tainarys sordida Burckhardt, 1987, Aphalaridae, formadora de agallas foliares del morfotipo 1; Calophya duvauae (Scott, 1882) y Calophya catillicola Burckhardt \& Basset, 2000, Calophyidae, formadoras de agallas foliares de los morfotipos 2 y 3 respectivamente. Estas especies fueron colectadas durante los meses de septiembre (solo adultos de Calophya duvauae) y diciembre (adultos de las tres especies). Del orden Lepidoptera se encontraron dos especies pertenecientes a Cecidosidae,

Tab. I. Especies de insectos inductores de agallas foliares (morfotipos $1,2,3$ ) y de tallo (morfotipos 4,5) en Schinus fasciculata (Anacardiaceae), Rio Cuarto, Argentina.

\begin{tabular}{|c|c|c|}
\hline Morfotipo & Familia & Especie \\
\hline 1 & Aphalaridae & Tainarys sordida Burckhardt \\
\hline $\begin{array}{l}2 \\
3\end{array}$ & Calophyidae & $\begin{array}{c}\text { Calophya duvauae (Scott) Burckhardt } \\
\text { Calophya catillicola Burckhardt \& Basset }\end{array}$ \\
\hline $\begin{array}{l}4 \\
5\end{array}$ & Cecidosidae & $\begin{array}{c}\text { Dicranoses congregatella } \text { Brèthes } \\
\text { Cecidoses eremita } \text { Curtis }\end{array}$ \\
\hline
\end{tabular}


Dicranoses congregatella Brèthes, 1916, cuyos adultos fueron colectados en noviembre y Cecidoses eremita Curtis, 1835, que fueron colectados en abril; ambas especies inductoras de agallas de tallos de los morfotipos 4 y 5 respectivamente. Las características exomorfológicas de cada morfotipo de agalla, foliar y de tallo, obtenido se detallan en la Tab. II.

El morfotipo 1 (Figs 1-4) consistió en la modificación de la totalidad de la lámina foliar como agalla, la que posee una forma irregular, por lo que no se consideraron las medidas de longitud y diámetro, fue originado por Tainarys sordida. En este único caso se encontraron más de una ninfa en el interior de una cámara, del mismo o de distintos estadios.

El morfotipo 2 (Figs 5-9) consistió en agallas cónicas inducidas por Calophya duvauae, situadas en la cara adaxial de las hojas y cuya coloración varió de acuerdo al desarrollo ontogenético del insecto inductor, desde verde en las primeras etapas, a rojo, y nuevamente verde antes de la emergencia de los adultos.

El morfotipo 3 (Figs 10-14) fue el único que consistió en agallas abiertas, en forma de depresiones circulares, inducidas por Calophya catillicola, Calophyidae, Hemiptera, cuyas ninfas permanecían con el lado dorsal de su cuerpo expuesto.

El morfotipo 4 (Figs 15-19) consistió en agallas cilíndricas en el tallo, inducidas por Dicranoses congregatella, Cecidosidae, Lepidoptera. Se encontraron agrupadas muy próximas entre si, formando con frecuencia un continuo sobre ramas de diferentes edades. La coloración varió desde verde, una vez que se desgarra la corteza del tallo, a rojo y nuevamente verde antes de la emergencia de los insectos adultos.

El morfotipo 5 (Figs 20-24) consistió en agallas esféricas en el tallo, inducidas por Cecidoses eremita, Cecidosidae, Lepidoptera. La coloración varió desde

Tab. II. Características de agallas foliares (morfotipos 1,2,3) y de tallo (morfotipos 4,5) en Schinus fasciculata (Anacardiaceae), Río Cuarto, Argentina. La agrupación indica el número de agallas por órgano afectado (A, abiertas; D, diámetro; DAO, dehiscencia apical por un opérculo; DO, diámetro en el opérculo; I, indehiscentes; I/C, insectos por cámara; L, longitud; $\mathrm{N}^{\circ} \mathrm{C}$, número de cámaras; $\mathrm{PC}$, parcialmente cerradas).

\begin{tabular}{|c|c|c|c|c|c|c|c|}
\hline Morfotipo & Ubicación en el órgano & Forma y descripción general & Dehiscencia & $\mathrm{N}^{\circ} \mathrm{C}$ & $\mathrm{I} / \mathrm{C}$ & $\begin{array}{l}\text { Dimensiones } \\
\text { Media }( \pm \mathrm{DE})\end{array}$ & Agrupación \\
\hline 1 & $\begin{array}{l}\text { Hojas jóvenes, } \\
\text { en ramas de 1-6 años }\end{array}$ & $\begin{array}{l}\text { Forma irregular, de color verde, con } \\
\text { vellosidades, blanda }\end{array}$ & $\mathrm{PC}$ & $1-2$ & $1-2$ & No se tomaron & $1-6$ \\
\hline 2 & $\begin{array}{l}\text { Haz de hojas, } \\
\text { en ramas de 1-2 años }\end{array}$ & $\begin{array}{c}\text { Cónica, de colores verde y rojo, } \\
\text { blanda }\end{array}$ & I & 1 & 1 & $\begin{array}{l}\mathrm{L}: 3.89 \pm 0.63 \mathrm{~mm} \\
\mathrm{D}: 3.26 \pm 0.52 \mathrm{~mm}\end{array}$ & $1-12$ \\
\hline 3 & $\begin{array}{l}\text { Haz de hojas, } \\
\text { en ramas de } 1-4 \text { años }\end{array}$ & $\begin{array}{l}\text { Depresión circular, de color amarillo } \\
\text { o verde, blanda }\end{array}$ & A & 1 & 1 & D: $0.73 \pm 0.25 \mathrm{~mm}$ & $1-5$ \\
\hline 4 & Ramas de 1-6 años & $\begin{array}{l}\text { Cilíndrica, de colores verde y } \\
\text { rojo, lignificada con excepción del } \\
\text { opérculo }\end{array}$ & DAO & 1 & 1 & $\begin{array}{l}\text { L: } 8.10 \pm 2.51 \mathrm{~mm} \\
\text { D: } 3.51 \pm 0.23 \mathrm{~mm} \\
\text { DO: } 0.88 \pm 0.08 \mathrm{~mm}\end{array}$ & $\begin{array}{l}\text { En número } \\
\text { variable }\end{array}$ \\
\hline 5 & Ramas de $1-5$ años & $\begin{array}{c}\text { Esférica, de colores verde y rojo, } \\
\text { lignificada }\end{array}$ & DAO & 1 & 1 & $\begin{array}{l}\text { D: } 13.23 \pm 0.65 \mathrm{~mm} \\
\text { DO: } 5.27 \pm 0.33 \mathrm{~mm}\end{array}$ & $1-11$ \\
\hline
\end{tabular}
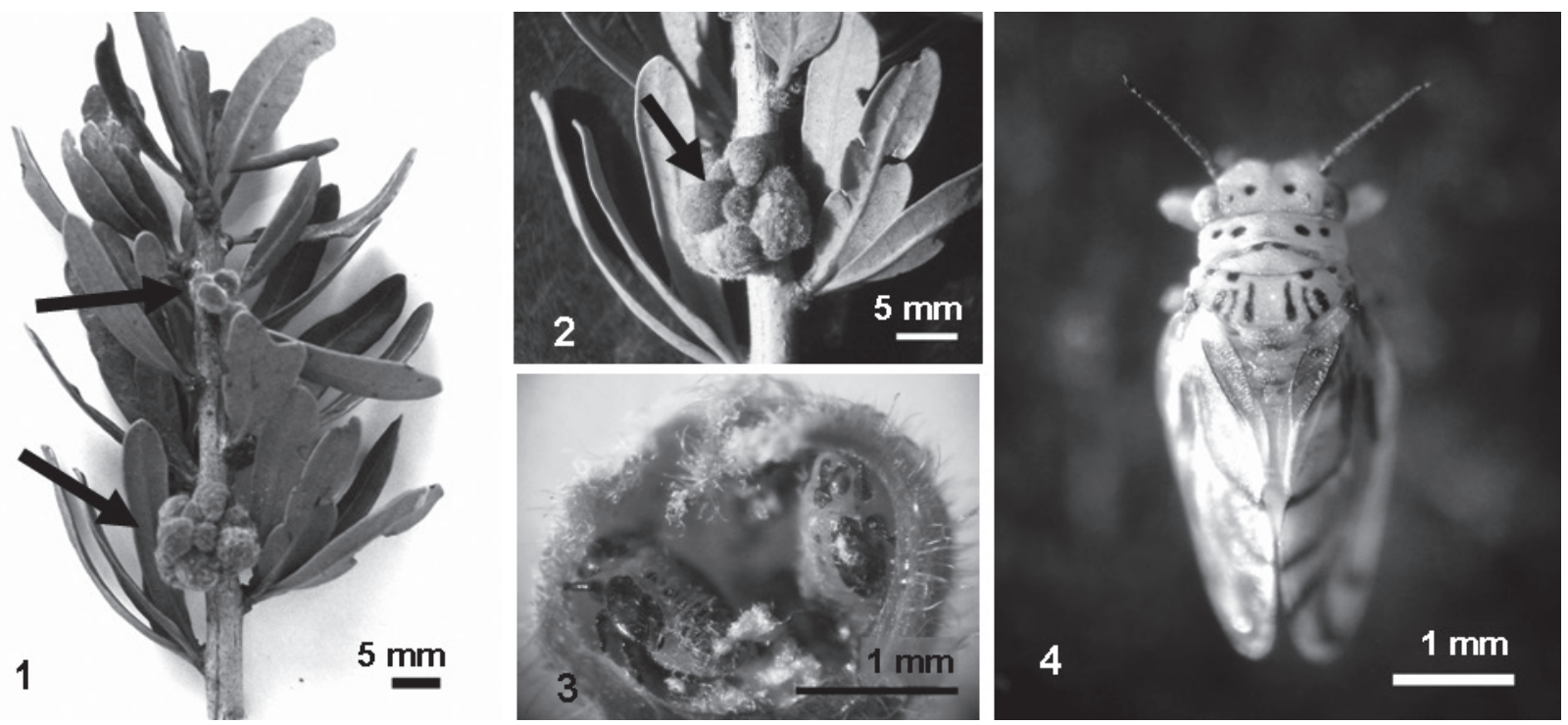

Figs 1-4. Agallas foliares del tipo 1: 1, rama de Schinus fasciculata con dos grupos de agallas; 2, braquiblasto con la totalidad de hojas modificadas en agallas; 3, ninfas en el interior de una cámara; 4, adulto de la especie inductora Tainarys sordida Burckhardt, 1987. 
verde claro y rojo, cuando las agallas fueron blandas, hasta verde oscuro al volverse leñosas antes de la emergencia de los adultos, momento en el cual el opérculo y una zona alrededor del mismo se secaron y se volvieron de color ocre.
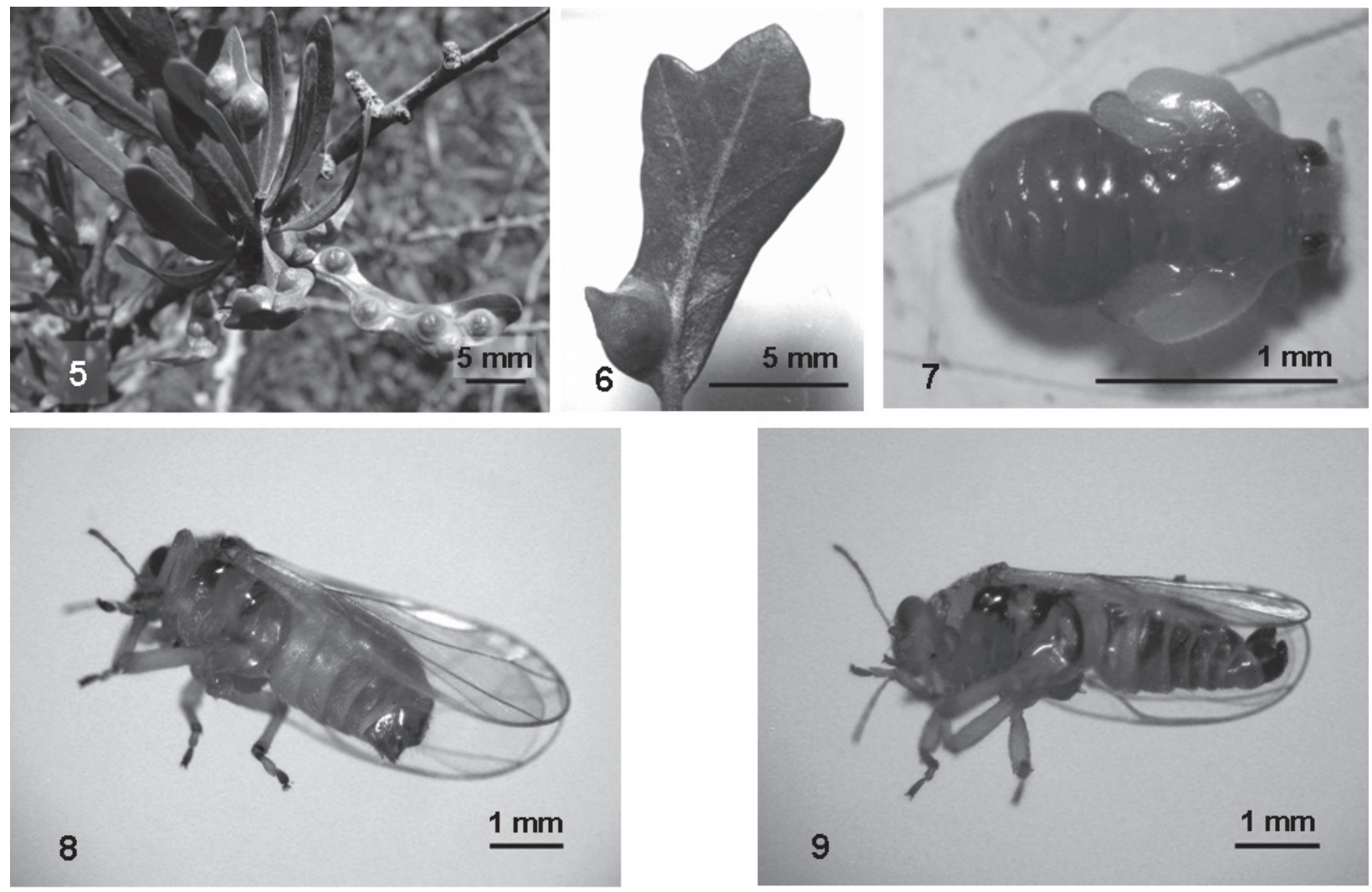

Figs 5-9. Agallas foliares del morfotipo 2: 5, braquiblasto de Schinus fasciculata con varias hojas que presentan agallas; 6, hoja con una agalla; 7, ninfa; 8, adulto hembra; 9, adulto macho de la especie inductora de agallas Calophya duvauae (Scott, 1882).
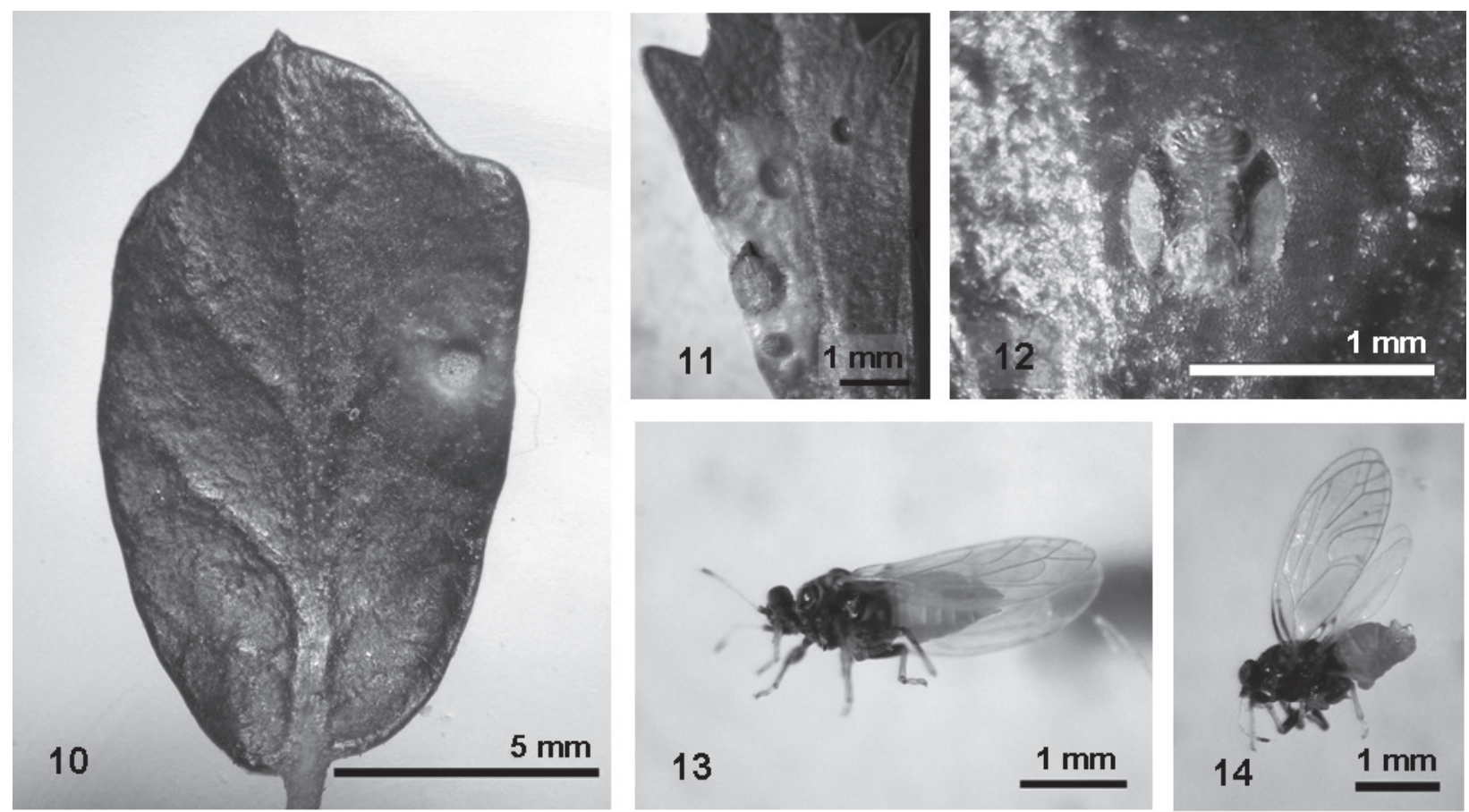

Figs 10-14. Agallas foliares del morfotipo 3: 10, hoja de Schinus fasciculata con una agalla; 11, hojas con dos agallas abandonadas y dos ninfas de distintos estadios; 12, ninfa; 13, adulto hembra; 14, adulto macho de la especie inductora de agallas Calophya catillicola Burckhard \& Basset, 2000. 

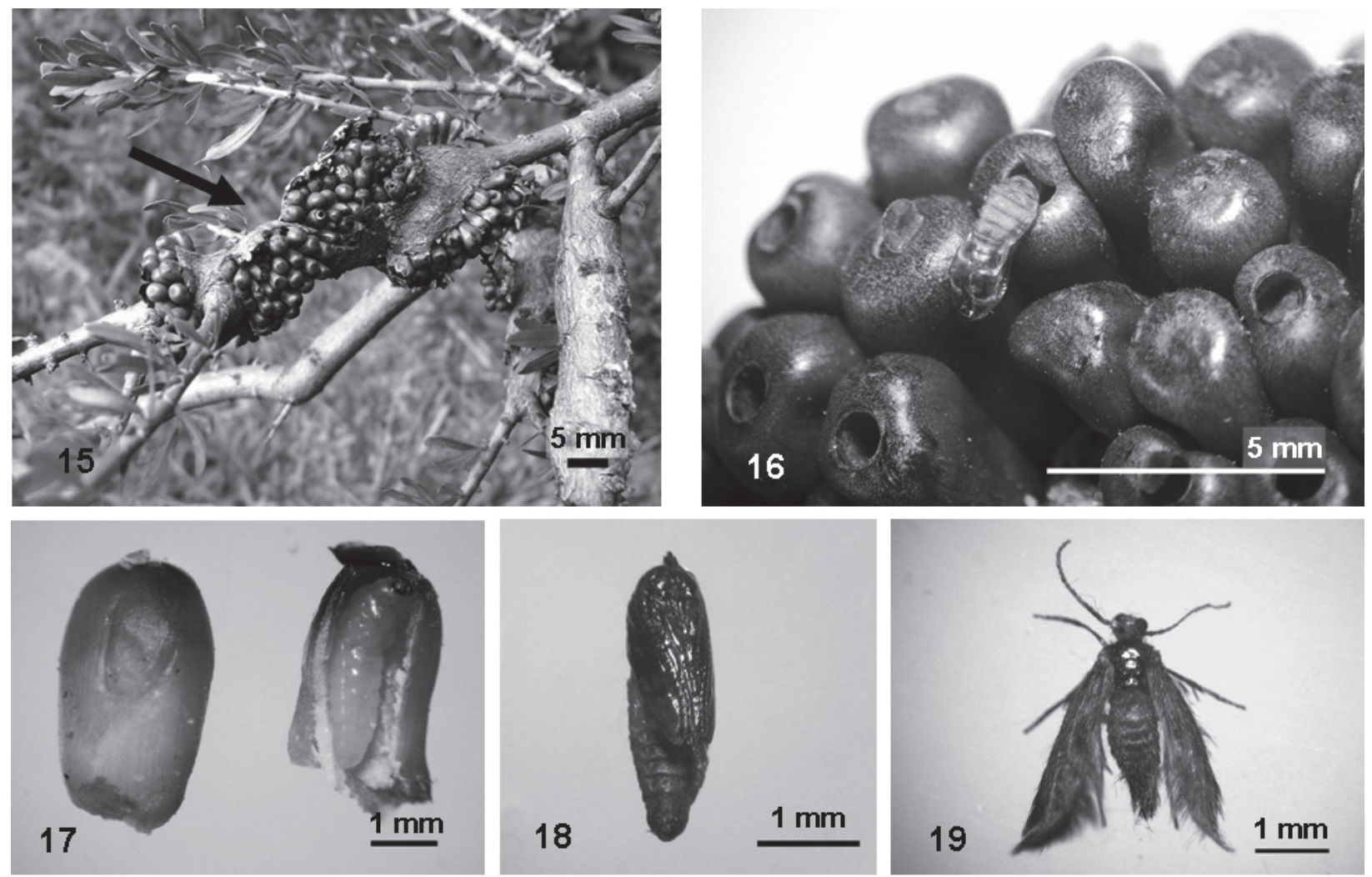

Figs 15-19. Agallas de tallo del morfotipo 4: 15, numerosas agallas agrupadas sobre una rama de Schinus fasciculata; 16, agallas con opérculos abiertos y una exuvia; 17, agallas con larvas en su interior; 18, pupa; 19, adulto de la especie inductora de agallas Dicranoses congregatella Brethes, 1916.
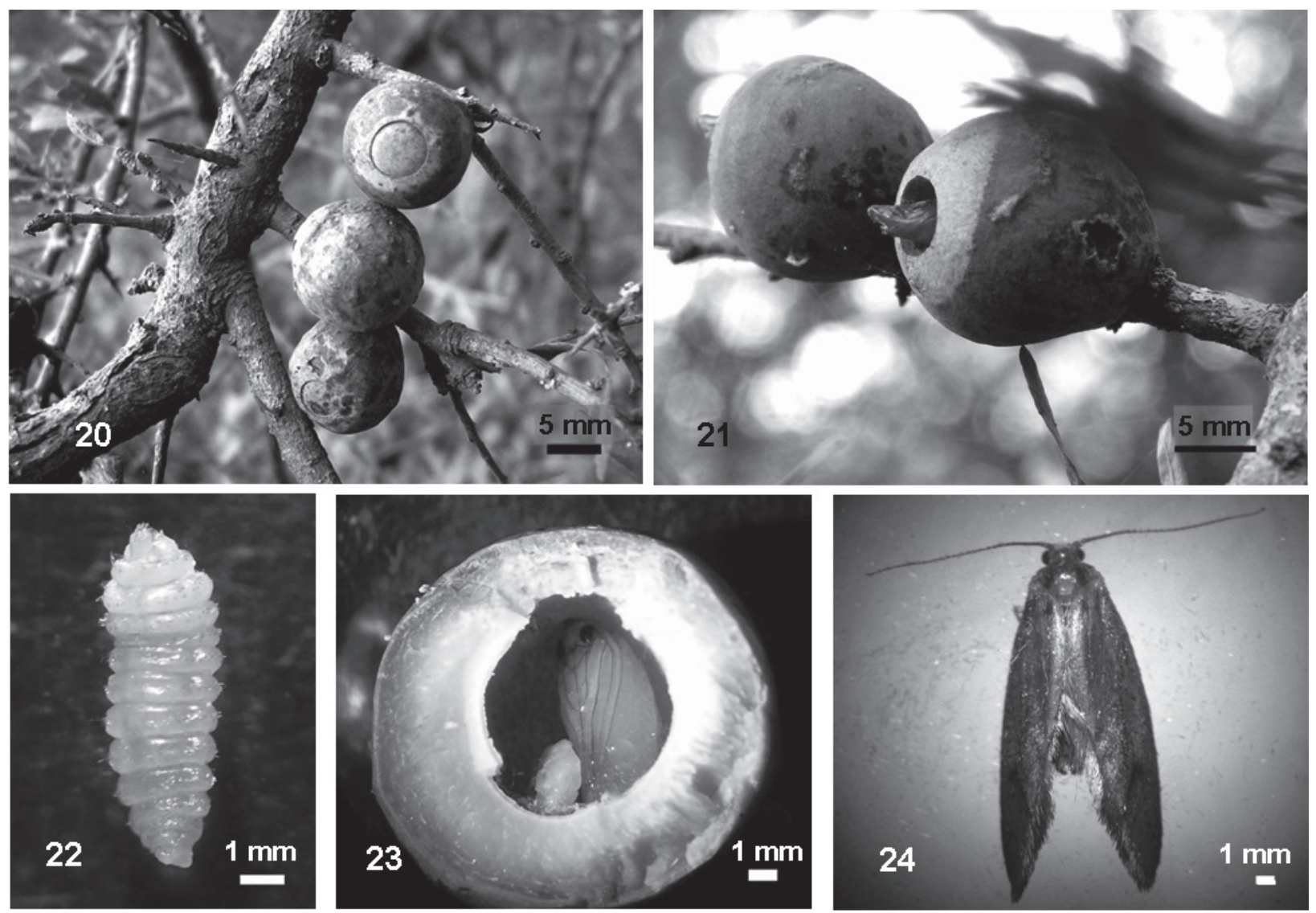

Figs 20-24. Agallas de tallo del morfotipo 5: 20, agallas maduras con opérculo diferenciados sobre ramas de Schinus fasciculata; 21, agallas abandonadas por los insectos, con exuvias en su interior; 22, larva; 23, pupa; 24, adulto de la especie inductora de agallas Cesidoses eremita Curtis, 1835. 


\section{DISCUSIÓN Y CONCLUSIONES}

En la región Neotropical, Cecidomyiidae (Diptera) es el grupo con mayor cantidad de especies galícolas, seguido por especies de los órdenes Hemiptera, Lepidoptera y Coleoptera (MAiA, 2006, 2012).

Los insectos galícolas registrados sobre Schinus fasciculata pertenecieron a cinco especies: Tainarys sordida (Aphalaridae), Calophya duvauae y Calophya catillicola (Calophyidae), pertenecientes al orden Hemiptera y Dicranoses congregatella y Cecidoses eremita, ambas especies de la familia Cesidosidae, orden Lepidoptera, sin registrarse especies de Cecidomyiidae, las que, sin embargo, han sido registradas en otras especies del género Schinus, sobre todo en Brasil (GAGNÉ, 1994)

Mientras que la riqueza de agallas por especie de planta varía usualmente entre uno y tres en la región árida del Chaco (Fernandes et al., 2002), en Schinus fasciculata se obtuvieron cinco morfotipos diferentes, esta importante riqueza de especies galícolas, asociada a una única especie de planta hospedadora, podría estar relacionada con la arquitectura compleja de la planta y con las características xéricas del ambiente de la ecorregión del Espinal; aunque FonseCA et al. (2006) y ARAúJo \& SANTOS (2009) proponen que diferentes especies de insectos galícolas responden de modo distinto al tamaño de la planta hospedadora, ya que durante la ontogenia de la planta ocurren una serie de transformaciones fisiológicas, como por ejemplo diferentes tasas de producción de compuestos de defensa como taninos y ligninas, la eficiencia fotosintética y el almacenamiento de nutrientes, que resultan en una mayor o menor preferencia de los insectos galícolas. Estas características podrían tener un papel más relevante sobre la riqueza y abundancia de insectos productores de agallas que la mera arquitectura de la planta.

De las especies de insectos galícolas registradas para el Espinal, sólo una de ellas, T. sordida, ha sido citada previamente sobre $S$. fasciculata con distribución en la provincia de Córdoba. La otra especie galícola de Taynaris registrada parasitando Schinus fasciculata, $T$. schinii Brèthes, a partir de material proveniente de Buenos Aires, originalmente fue hecha sobre Schinus dependens Ortega, un actual sinónimo de Schinus johnstonii F. A. Barkley (y no de S. fasciculata), una especie de distribución esencialmente patagónica, que alcanza en su distribución norte a la provincia de Buenos Aires. Los registros previos de Calophya parasitando sobre Schinus fasciculata, tales como Calophya catillicola, Calophya gallifex (Kieffer \& Jörgensen) y Calophya orbicola Burckhardt, fueron hechos con material pertenecientes a otras regiones biogeográficas (BURCKHARDT \& BASSET, 2000), en particular la provincia biogeográfica del Monte, de la Zona de Transición Sudamericana, por lo que consideramos que este estudio resulta un importante antecedente para la provincia de Córdoba en el centro de Argentina, contribuyendo al conocimiento de la riqueza de los insectos inductores de agallas en la provincia biogeográfica de La Pampa, perteneciente a la subregión Chaqueña.

Las especies galícolas difirieron en cuanto al momento de emergencia de sus estados adultos, mientras que los adultos de T. sordida, C. duvanae, y C. catillicola emergieron en distintos momentos durante la realización del estudio, los adultos de $D$. congregatella emergieron en noviembre y los adultos de $C$. eremita emergieron en abril y mayo, coincidiendo, esto último, con lo observado por FernÁndeZ \& D'AmBrogio (2001).

Cada una de las especies de insectos galícolas indujo distintos morfotipos de agallas, y los cinco morfotipos de agallas resultaron diferentes, fácilmente identificables y únicos para cada especie de insecto inductor. El morfotipo 1, originado por $T$. sordida, fue muy semejante al descrito por Lizer y TRelLes \& Molde (1945) originado por T. schinii Brèthes, sobre S. polygama. Posteriormente BURCKHARDT \& BASSET (2000) mencionaron estas agallas, originadas por $T$. sordida en $S$. fasciculata, como enrollamientos irregulares de las hojas, pero no reportaron la presencia de tricomas. La descripción del morfotipo 2 , inducido por $C$. duvauae, coincidió totalmente con la realizada por LizER Y TRELLES \& Molle (1945) sobre S. polygama. BURCKHARDT \& BASSET (2000) describen estas agallas como situadas sobre la cara abaxial de las hojas de Schinus fasciculata. La discrepancia con los resultados obtenidos en este trabajo puede deberse a que BURCKHARDT \& BASSET (2000) siguieron la descripción realizada por Scott (1882) y Houard (1933), quienes confunden la ubicación de las agallas, como lo expresan Lizer \& Trelles Molle (1945). El morfotipo 3, originado por C. catillicola, consistió en agallas abiertas situadas sobre hojas jóvenes, coincidiendo con la descripción de BURCKHARDT \& BASSET (2000). El morfotipo 4, inducido por D. congregatella, consistió en agallas cilíndricas dispuestas de forma agrupada sobre los tallos, coincidiendo con COSTA Lima (1945) sobre S. polygama, para Brasil. La otra especie de Dicranoses, D. capsulifex Kieffer and Jörgensen, citada parasitando Schinus fasciculata en Mendoza, oeste de Argentina, produce agallas muy diferentes, de tipo foliar y con paredes mucho más delgadas. El último morfotipo de agalla, inducido por $C$. eremita, consistió en estructuras esféricas situadas en los tallos, siendo muy semejantes a las descritas por FERNÁNDEZ \& D'AMBROGIO (2001) sobre $S$. marchandii, excepto porque éstas agallas no se ubicaron en ramas de un año, presentaron otra coloración, se agruparon en menor número y tuvieron un menor diámetro.

Agradecimientos. A Daniel Burckhardt (Naturhistorisches Museum, Suiza) y Adriana Chalup (Instituto Miguel Lillo, Argentina), por la identificación de los imagos. A la Secretaría de Ciencia y Técnica de la Universidad Nacional de Río Cuarto, por el subsidio otorgado para la realización de este trabajo.

\section{REFERENCIAS BIBLIOGRÁFICAS}

Arana, M. D. \& Bianco, A. C. 2011. Características ambientales del centro de la Argentina, pp: 8-18. En Helechos y Licofitas del centro de la Argentina. Río Cuarto, Editorial UNRC. 84p.

Araúso, W. S. \& dos Santos, B. B. 2009. Efeitos da sazonalidade e do tamanho da planta hospedeira na abundância de galhas de Cecidomyiidae (Diptera) em Piper arboreum (Piperaceae). Revista Brasileira de Entomologia 53(2):300-303. 
Barkley, F. A. 1957. A study of Schinus L. Lilloa 22:5-110.

Barrancos, M. L.; Moncaglieri, R. \& FarJi-Brener, A. 2008. Infección por agallas y producción de inflorescencias en el arbusto patagónico Schinus patagonicus. Ecología Austral 18:133-137.

Burckhardt, D. 2004a. Calophyidae, pp. 329-330. In: Cordo, H. A.; Logarzo, G.; Braun, K. \& Di Dorio, O. R. eds. Catálogo de insectos fitófagos de la Argentina y sus plantas asociadas. Buenos Aires, Sociedad Entomológica Argentina. 734p.

2004b. Psyllidae, pp. 388-392. In: Cordo, H. A.; Logarzo, G.; Braun, K. \& Di Iorio, O. R. eds. Catálogo de insectos fitófagos de la Argentina y sus plantas asociadas. Buenos Aires, Sociedad Entomológica Argentina. 734p.

BurckHARDT, D. \& BASSET, Y. 2000. The jumping plant-lice (Hemiptera, Psylloidea) associated with Schinus (Anacardiaceae): systematics, biogeography and host plant relationships. Journal of Natural History 34:57-155.

Cuevas-Reyes, P.; Quesada, M.; Hanson, P.; Dirzo, R. \& Oyama, K. 2004. Diversity of gall-inducing insects in a Mexican tropical dry forest: the importance of plant species richness, life-forms, host plant age and plant density. Journal of Ecology 92:707-716.

Costa Lima, A. DA. 1945. Insetos do Brasil. Tomo 5. Lepidoptera. Serie didáctica 4. Rio de Janeiro, Escola Nacional de Agronomia. 379p.

Espírito-Santo, M. M. \& Fernandes, G. W. 2007. How Many Species of Gall-Inducing Insects Are There on Earth, and Where Are They? Annals of the Entomological Society of America 100(2):95-99.

Fernandes, G. W.; Coelho, M. S. \& Santos, J. C. 2014. Cap. 1: Neotropical Insect Galls: Status of Knowledge and Perspectives, pp 1-15. En: Fernandes, G. W. \& Santos, J. C. (Ed.) Neotropical Insect Galls. New York, Springer. 550 p.

Fernandes, G. W. \& Price, W. P. 1988. Biogeographical gradients in galling species richness. Tests of hypotheses. Oecologia 76:161-167.

1992. The adaptive significance of insect gall distribution: survivorship of species in xeric and mesic habitats. Oecologia 90:14-20.

Fernandes, G. W.; Varela, O.; Bucher, E. H.; Chani, J. M.; Echevarria, A. L.; Espírito Santo, M. M.; Lima, J.; Negreiros D. \& Saravia Toledo, C. 2002. Gall-forming insects on woody and herbaceous plant species of the semi-arid chaco forest, Argentina. Lundiana 3(1):61-66

Fernandes, G.W.; Carneiro, M.A.A. \& Isaias, R.M.S. 2011. Gall-inducing insects: from anatomy to biodiversity, pp 369-395. In: PANIzZI, A.R. \& PARRA, J.R.P. (eds) Insect bioecology and nutrition for integrated pest management. Boca Raton, CRC Press. 750p.

Fernández, S. \& D’Ambrogio, A. 2001. Cecidios en una Anacardiácea patagónica. Boletín de la Sociedad Argentina de Botánica 36(34):243-251.

Fonseca, C. R.; Fleck, T. \& Fernandes, G. W. 2006. Processes driving ontogenetic sucession of galls in canopy tree. Biotropica 38:514-521
Gagné, R. J. 1994. The gall midges of the Neotropical region. New York, Cornell University Press. 352p.

Houard, C. 1933. Les zoocécidies des plantes de l'Amérique du Sul et de l' Amérique Centrale. Paris, Hermann et Cie. 549 p.

Jörgensen, P. 1917. Zoocecidios argentinos. Physis 13(3):1-29.

Lara, A. C. F.; Fernandes, G. W. \& Gonçalves-Alvim, S. J. 2002. Tests of hypotheses on patterns of gall distribution along an altitudinal gradient. Tropical Zoology 15:219-232.

Lizer y Trelles, C. A. \& Molle, C. C. 1945. Estructura anatómica de filocecidias neotropicas. Lilloa 11:153-187.

MaiA, V.C. 2006. Galls of Hemiptera, Lepidoptera and Thysanoptera from Central and South America. Publicações Avulsas do Museu Nacional 110: 3-22.

MAIA, V.C. 2012. Coleopterous galls from the Neotropical region. Papéis Avulsos de Zoologia 52(15): 175-184.

Mani, M. S. 1964. The ecology of plant galls. The Hague, W. Junk. 434p.

Martínez Carretero, E. 2009. Flora de Mendoza. Multequina 18(2):129176.

Morrone, J. J. 2004. La Zona de Transición Sudamericana: caracterización y relevancia evolutiva. Acta Entomológica Chilena 28:41-50.

Nieves-Aldrey, J. L. 1998. Insectos que inducen la formación de agallas en plantas: una fascinante interacción ecológica y evolutiva. Boletín Sociedad Entomológica Aragonesa 23:3-12.

Pastrana, J. A.; Di Iorio, O. R.; Navarro, F.; Chalup, A. \& Villagrán, M. E. 2004. Lepidoptera. In: Cordo, H. A.; Logarzo, G.; Braun, K. \& Di Iorio, O. R. eds. Catálogo de insectos fitófagos de la Argentina y sus plantas asociadas. Buenos Aires, Sociedad Entomológica Argentina. 734p.

Price, P. W.; Fernandes, G. W.; Lara, A. C. F.; Brawn, J.; Barrios, H.; Wright, M. G.; Ribeiro, S. P. \& Rothcliff, N. 1998. Global patterns in local number of insect galling species. Journal of Biogeography 25:581-591.

RAMAN, A. 2007. Insect-induced plant galls of India: unresolved questions. Current Science 6(92):748-757.

Santos, J.C.; Carneiro, M. A. A. \& Fernandes, G. W. 2012. Insetos galhadores neotropicais: diversidade e ecologia evolutiva dos herbívoros mais sofisticados da natureza, pp 183-199. In: Del Claro, K. \& Torezan-Silingardi, H. M. eds. Ecologia das Interações Plantas-Animais: Uma Abordagem Ecológico-Evolutiva. Rio de Janeiro, Technical Books. 333p.

Shorthouse, J. D.; Wool, D. \& Raman, A. 2005. Gall-inducing insectsNature's most sophisticated herbivores. Basic and Applied Ecology 6:407-411.

Vieira da Costa, F.; Fagundes, M. \& de Siqueira Neves, F. 2010. Arquitetura da planta e diversidade de galhas associadas à Copaifera langsdorffii (Fabaceae). Ecología Austral 20:9-17. 